\title{
Caminhos da avaliação da aprendizagem ativa: visão do estudante de medicina
}

\author{
Elza Higa, Haydée Moreira, Osni Pinheiro, \\ Silvia Tonhom, Maria Helena de Carvalho \\ \& Luzmarina Braccialli
}

\begin{abstract}
Resumo:
As instituições de ensino superior, no Brasil, a partir da década de 1980, no âmbito de sua autonomia concedida pelo Sistema Nacional de Avaliação da Educação Superior (SINAES) agregam a avaliação dos estudantes por diferentes formas e instrumentos utlizados. A avaliação contempla estratégias pedagógicas e pode contribuir para ajustes requeridos no processo de construção do conhecimento. O objetivo dessa pesquisa foi analisar a representação social do estudante de medicina sobre o processo de ensino-aprendizagem e os recursos educacionais estruturados na Unidade Educacional Sistematizada (UES). A pesquisa teve abordagem qualitativa fundamentada na Teoria das Representações Sociais, analisada pela técnica do Discurso do Sujeito Coletivo e participaram 16 estudantes do quarto ano de medicina. Os resultados indicaram oito ideias centrais: impetrou os recursos de aprendizagem; reflexões clínicas e terapêuticas; correlação teórica e prática eficiente e eficaz; teoria e prática às vezes desafinadas; fidelidade aos passos da tutoria; falta preparo do preceptor e do tutor e fortalezas e fragilidades dos recursos educacionais. A avaliação dos estudantes destacou os pontos fortes e frágeis do processo ensino e aprendizagem, bem como dos recursos educacionais e forneceu subsídios para as adequações necessárias à melhoria proposta educacional da UES e curricular.
\end{abstract}

Palavras-chave:

aprendizagem; avaliação educacional; educação médica. 


\title{
Roads of active learning assessment: the medical student's vision
}

\begin{abstract}
The institutions of university education, in Brazil, starting from 1980s, in the ambit of its anatomy granted by the National System of higher education evaluation (SINAES) join the evaluation of students by different forms and instruments used. The evaluation considers pedagogical strategies and can contribute to the adjustments required in the construction process of the knowledge. The objective of this research was to analyze the social representation of the medical student about the process of teaching-learning and the educational resources built into the Systematized Educational Unit (UES). The research has had a qualitative approach based in the Theory of Social Representations, analyzed by the technique of the Collective Subject Discourse and 16 students from the fourth year of medicine. The results indicated eight central ideas: implored learning resources; clinical and therapeutic reflections; efficient and effective theoretical and practical correlation; theory and practice sometimes out of tune, fidelity to the steps of tutoring; lack of preparation of the preceptor and the tutor and strengths and weaknesses of the educational resources. The evaluation of the students highlighted the strong and fragile points of the teaching-learning process, as well as of educational resources and provided subsidies for adjustments needed to improve the educational proposal of the UES and curricular.
\end{abstract}

Keywords: learning; educational evaluation; medical education

\section{Les chemins de l'évaluation de l'apprentissage actif: la vision de l'étudiant en médecine}

Résumé: Depuis les années 1980, au Brésil, les établissements d'enseignement supérieur, dans le cadre de leur autonomie accordée par le Système national d'évaluation de l'enseignement supérieur (SINAES), agrègent l'évaluation des étudiants par différentes formes et instruments. L'évaluation envisage des stratégies pédagogiques et peut contribuer aux ajustements requis dans le processus de construction des connaissances. L'objectif de cette recherche était d'analyser la représentation sociale de l'étudiant en médecine sur le processus d'enseignementapprentissage et les ressources pédagogiques structurées dans l' Unité éducative systématisée (UES). La recherche a eu une approche qualitative basée sur la Théorie des Représentations Sociales, analysée par la technique du Discours du Sujet Collectif et avec la participation de 16 étudiants de la quatrième année de médecine. Les résultats ont indiqué huit idées centrales: il a introduit les ressources d'apprentissage; réflexions cliniques et thérapeutiques; corrélation théorique et pratique efficace et efficiente; théorie et pratique, parfois désaccordées; la fidélité aux étapes du tutorat; manque de préparation du précepteur et du tuteur et forces et faiblesses des ressources éducatives. L'évaluation des étudiants a mis en évidence les points forts et les points faibles du processus d'enseignement / d'apprentissage ainsi que les ressources pédagogiques et a fourni des subventions pour les ajustements nécessaires à l'amélioration de l'éducation et de l'enseignement de l'UES et de son programme d'études.

Mots-clés: apprentissage; évaluation éducative; éducation médicale.

\section{Caminos de la evaluación del aprendizaje activo: la visión del estudiante de medicina}

Resumen: Las instituciones de enseñanza universitária, en Brasil, a partir de la década de 1980, en el ámbito de su anatomia concedida por el Sistema Nacional de Evaluación de la Educación Universitária (SINAES) agregan la evaluación de los estudiantes por distintas formas e instrumentos utilizados. La evaluación contempla estratégias pedagógicas y puede contribuir para ajustes requeridos en el proceso de construcción del conocimiento. El objetivo de esa investigación fue analisar la representación social del estudiante de medicina sobre el proceso de enseñanzaaprendizaje y los recursos educacionales estruturados en la Unidad Educativa Sistematizada (UES). La investigación ha tenido un abordaje cualitativo fundamentado en la Teoria de Representaciones Sociales, analisada por la técnica del Discurso del Sujeto Colectivo y han participado 16 estudiantes del cuarto año de medicina. Los resultados indicarón ocho ideas centrales: imploróse los recursos de aprendizaje; reflexiones clínicas y terapéuticas; correlación teórica y práctica eficiente y eficaz; teoria y práctica a veces desafinadas, fidelidad a los pasos de la tutoria; falta de preparo del preceptor y del tutor y fortalezas y fragilidades de los recursos educacionales. La evaluación de los estudiantes destacó los puntos fuertes y frágiles del proceso enseñanza-aprendizaje, bien como de los recursos educacionales y proporcionó subsídios para las adecuaciones necesárias a la mejoría propuesta educacional de la UES y curricular.

Palabras-clave: aprendizaje; evaluación educacional; educación médica. 
Higa, Moreira, Pinheiro, Tonhom, Carvalho \& Braccialli: Caminhos da avaliação da aprendizagem ativa

\section{Introdução}

No contexto das Instituições de Ensino Superior (IES), a avaliação deve contemplar estratégias pedagógicas que regulem e supervisionem o ensino e a aprendizagem com o propósito de melhorar sua qualidade educacional (Antunes, 2017).

Felix, Bertolin \& Polidori (2017), ao abordarem sobre a avaliação no ensino superior, apontam a importância da melhoria na qualidade e regulação, tanto para os países da América do Sul quanto para os países europeus. Nesse cenário, a comunidade europeia, preocupada com a qualidade do ensino superior e com o descompasso entre instituições, profissionais e governos, introduz novos mecanismos de avaliação e acreditação.

O Brasil, também atento à importância da qualidade da avaliação no ensino superior, institui, a partir da década de 1980, o Sistema Nacional de Avaliação da Educação Superior (SINAES), coordenado pelo Instituto Nacional de Ensino e Pesquisa (INEP) e Ministério de Educação e Cultura (MEC). No âmbito de sua autonomia, agrega a avaliação dos estudantes por diferentes formas e instrumentos (Bettencourt \& Sá Earp, 2017). Destaca-se, dentre esses instrumentos, o Exame Nacional de Desempenho do Estudante (ENADE), que gera indicadores de qualidade dos cursos e subsidia o MEC nas ações de regulação e credenciamento das Instituições de Ensino Superior (IES) (Felix, Bertolin \& Polidori, 2017).

Na área da Saúde, os cursos fizeram adequações em seus processos de ensino e aprendizagem e, consequentement, na avaliação. Essas reformulações foram impulsionadas pelas Diretrizes Curriculares Nacionais (DCN) de 2001. A Faculdade de Medicina de Marília, pioneira no Brasil na implantação de metodologia de aprendizagem ativa nos Cursos de Medicina e Enfermagem (Higa, Taipeiro, Aguilar-da-Silva, CarvaIho, Tonhom, \& Hafner, 2016), estruturou seu planejamento educacional, considerando a inserção dos estudantes nos cenários de prática profissional e, concomitantemente, na Unidade Educacional Sistematizada (UES) desde a primeira série. Essa organização visou contribuir com o desenvolvimento integrado dos recursos cognitivos, psicomotores e afetivos, ancorados pelo olhar ampliado das necessidades de saúde dos indivíduos e suas famílias, com enfoque nos aspectos biopsicossociais e no estabelecimento da competência médica (Faculdade de Medicina de Marília, 2016).

$\mathrm{Na}$ contemporaneidade, essa lógica curricular nasce da necessidade de adequações na formação dos profissionais tanto para melhorar o cuidado à saúde quanto para atualização e manejo das novas tecnologias educacionais (Leon \& Onófrio, 2015).

Assim, o desenvolvimento curricular na Famema passou a ser permanente e de responsabilidade de todos os envolvidos no processo. A UES, objeto de estudo desta pesquisa, utiliza a metodologia da Aprendizagem Baseada em Problemas (ABP) e 
oferece atividades de experimentação prática em laboratórios (Faculdade de Medicina de Marília, 2014).

$\mathrm{Na} 4^{a}$ série, a partir do alicerce desses conhecimentos, construídos gradativamente nos anos anteriores, e dando continuidade à aprendizagem específica, os estudantes buscam consolidar e aplicar o entendimento do processo saúde-doença, tendo em vista as intervenções necessárias na lógica da integralidade do cuidado (Faculdade de Medicina de Marília, 2016). Nesse contexto, eles têm a oportunidade de desenvolver também os aspectos fundamentais para a qualidade do cuidado, como respeito, autonomia, consciência cidadã, trabalho em equipe e gestão compartilhada do trabalho, necessários à construção da competência do médico (Faculdade de Medicina de Marília, 2016).

Nessa estratégia organizacional, as atividades são desenvolvidas em diferentes cenários do Sistema Único de Saúde (SUS), nos quais o estudante pode desenvolver um olhar crítico e reflexivo quanto a proposta, organização, resolutividade e fragilidades do SUS. Possibilita, ainda, o trabalho multi e interprofissional, que se exercita na prática, nas relações com o outro, com a equipe e com a comunidade. Os estudantes atuam, então, desde a promoção à saúde até a sua recuperação e reabilitação, a fim de desenvolver os recursos esperados para a formação de um profissional competente (Faculdade de Medicina de Marília, 2016).

Na mesma lógica das séries anteriores, por meio de metodologias de aprendizagem ativa, o estudante transita por cenários diversificados, incluindo a Estratégia Saúde da Família (ESF) e Unidades Hospitalares (UH). Na 4a série, a estrutura para a aprendizagem envolve Unidade de Prática Profissional (UPP4) - onde a vivência da prática é desenvolvida sob a supervisão dos profissionais das equipes nas Unidades Básicas de Saúde (UBS) e professores da universidade, Cirurgia Ambulatorial, e Ambulatórios de Especialidades. O Laboratório de Prática Profissional (LPP) apoia o desenvolvimento de habilidades necessárias aos cenários de prática (Faculdade de Medicina de Marília, 2016).

A reflexão sobre a realidade é desenvolvida por meio do ciclo pedagógico, em que ocorre a discussão sobre uma situação-problema, com identificação de necessidades de aprendizagem e busca na literatura para responder às lacunas necessárias à resolução dos problemas encontrados. Esse movimento é registrado no portfólio reflexivo e orientado pelo professor da UPP4. As reuniões anatomoclínicas muito contribuem para o desenvolvimento das habilidades em apresentação de casos e na organização dos conhecimentos (Faculdade de Medicina de Marília, 2016).

A UES está constituída pelas apresentações de casos clínicos, sob a forma de tutorias, além das conferências e consultorias. Exige-se, além, um trabalho de iniciação científica, com a elaboração de um projeto de pesquisa, cujo problema esteja vinculado à prática vivenciada pelo estudante, devidamente orientado por um docente (Faculdade de Medicina de Marília, 2016). 
Higa, Moreira, Pinheiro, Tonhom, Carvalho \& Braccialli: Caminhos da avaliação da aprendizagem ativa

Nas UES, frequentemente, trabalha-se com situações-problema, em que são apresentados aos estudantes informações, conhecimentos e experiências que serão aplicados no cotidiano da vida profissional. Nessa metodologia, a aprendizagem pode ser potencializada por sucessivas aproximações, que oportunizam apreender e reconstruir os conhecimentos (Barros \& Tamblyn, 1980; Castilho, Higa, Otani, \& Soares, 2017).

Os autores apontam que a ABP contribui para que o estudante desenvolva a capacidade de aprender a aprender em diferentes situações, sendo corresponsável pela própria educação e avaliação. Nesse caminho, o estudante vai desenvolvendo sua capacidade avaliativa, que subsidia a percepção de suas fragilidades, fortalezas e necessidades de aprendizagens, sendo necessários recursos institucionais, como livros, monografias, laboratório, biblioteca e audiovisuais. (Barros \& Tamblyn, 1980; Castilho et al., 2017).

No planejamento educacional da $4^{a}$ série da Famema, estão incluídos, ainda, os recursos educacionais em complementação e apoio a todas as atividades de aprendizagem desenvolvidas, a saber: Biblioteca, Laboratório Morfofuncional, Consultorias, conferências e/ou outros (Faculdade de Medicina de Marília, 2016).

A avaliação do Curso de Medicina, por ser formativa e somativa, contempla o estudante, o professor, a Unidade Educacional Sistematizada e os Recursos Educacionais. Para isso, são utilizados instrumentos que permitem ao professor coletar informações sobre a capacidade de aprendizado dos estudantes, medida, principalmente pela competência deles para resolver problemas e instrumentalizar o conhecimento para a tomada de decisões, bem como para avaliar, concomitantemente, o professor e o próprio planejamento educacional (Faculdade de Medicina de Marília, 2015).

Para a avaliação formativa, um dos instrumentos utilizados é o Formato Cinco (F5), constituído por seis campos: 1. Proposta da Unidade Educacional Sistematizada; 2. Processo de ensino-aprendizagem; 3. Problemas; 4. Recursos educacionais; 5. Conferências; 6. Comentários adicionais/sugestões/recomendações. (Castilho et al., 2017; Faculdade de Medicina de Marília, 2015).

Este estudo se propõe a avaliar a representação social do estudante sobre a organização da Unidade Educacional Sistematizada (UES), por meio do Formato Cinco (F5), no que se refere aos campos dois e quatro, cujas especificações estão nos resultados, visto que há necessidade de compreender como os estudantes estão avaliando a estrutura organizacional da UES por meio do F5 (Faculdade de Medicina de Marília, 2015). Sua finalidade é conseguir subsídios à gestão curricular das escolas médicas, tendo em vista os ajustes que se fizerem necessários. A pergunta direcionadora desta pesquisa foi assim estabelecida - como os estudantes estão avaliando o processo de ensino-aprendizagem, quais os recursos educacionais utilizados na UES e que são representados, em seus discursos, por meio do F5? O objetivo é Analisar a representação social do estudante de medicina sobre o processo de ensino-aprendizagem e os recursos educacionais estruturados na UES. 


\section{Aspectos metodológicos}

Pesquisa ex-post-facto, qualitativa, fundamentada nos pressupostos da Teoria de Representações Sociais (TRS), retomada por Serge Moscovici em 1960, em La psychanalise, son image et son public, sendo significativa para estudos nas ciências sociais e na psicologia pela interpretação das representações mentais expressas pelo fenômeno real estudado (Duran, 2012; (Moscovici, 2009; Higa, Moreira, Pinheiro, Tonhom, Carvalho, \& Braccialli, 2017; Reis \& Bellini, 2013).

No ponto de vista psicossociológico, proposto por Moscovici, as pessoas se comunicam como agentes ativos, acionam as informações e revelam suas representações para solucionar conjecturas específicas. As representações sociais são consideradas um arcabouço de saberes, utilizado para apresentar a realidade de modo mais compreensível (Moscovici, 2009; Higa et al., 2017).

A Teoria das Representações Sociais acomoda quatro características essenciais: 1) concebe um objeto, fenômeno e/ou um sujeito; 2) pressupõe haver uma associação entre o simbolismo e a significação dos objetos; 3) a representação conforma um modo de distinguir e formar modelos dos objetos por meio de estrutura linguística; 4) as pessoas realizam ponderações e definem características do conhecimento de acordo com seu contexto social (Moscovici, 2009; Reis \& Bellini, 2013). Dessa maneira, esta teoria proporciona um novo olhar dirigido aos saberes produzidos no cotidiano da sociedade contemporânea.

Assim, na visão dos estudiosos, o compartilhamento das representações sobre um mesmo fenômeno, numa determinada realidade, entre os diferentes indivíduos e grupos sociais, constrói uma representação social. Ana Maria Cavalcanti Lefèvre e Fernando Lefèvre, na década de 90, tendo como parâmetro os pressupostos da Teoria das Representações Sociais, retomaram essas representações expressas em circunstâncias vivenciadas em uma determinada sociedade e cultura e estruturaram um método de análise de dados denominado Discurso do Sujeito Coletivo (DSC) (Lefèvre \& Lefèvre, 2012; Figueiredo, Chiari, \& Goulart, 2013).

O DSC é organizado pelas seguintes figuras metodológicas: Expressões-chave $(\mathrm{ECH})$, Ideias Centrais (IC) e Ancoragem (AC). As ECH são trechos dos depoimentos que caracterizam o sentido do dado obtido; as IC representam a marca do discurso, a síntese e o resgate da descrição do sentido do depoimento, sendo a $A C$ a expressão de uma determinada teoria que o autor do discurso professa. O DSC deve ser redigido na primeira pessoa do singular, reunindo os discursos que têm a mesma ideia central, representando a fala de todos os participantes (Lefèvre \& Lefèvre, 2005b, 2012).

Nesta pesquisa, a sequência de passos até chegar à síntese dos discursos obedeceu à seguinte ordem: 1) Análise dos depoimentos coletados na leitura dos formatos selecionados a partir da qualificação da avaliação, de modo satisfatório ou 
Higa, Moreira, Pinheiro, Tonhom, Carvalho \& Braccialli: Caminhos da avaliação da aprendizagem ativa

insatisfatório; 2) Análise da resposta de cada um em particular, com destaque para as expressões-chave; 3) consideração das ideias centrais de cada resposta; 4) aglomeração e análise de todos os conjuntos análogos entre ideia central e expressões-chave; 5) elaboração dos discursos do sujeito coletivo de cada ideia central (Higa et al., 2017; Lefèvre \& Lefèvre, 2005a, 2005b)

Considerando os aspectos éticos e legais, contidos na Resolução n 466, de 12 de dezembro de 2012 do Conselho Nacional e Saúde Brasil (2013), a pesquisa foi autorizada pelo Comitê de Ética em Pesquisa - CEP envolvendo Seres Humanos, da Faculdade de Medicina de Marília - Famema, sob o parecer $n^{\circ}$. 1.210.110.

\section{Resultados}

A análise das respostas obtidas, a partir da avaliação dos itens dois e quatro do Formato cinco (F5) apresenta oito ideias centrais acompanhadas de seus respectivos discursos.

No que se refere ao processo de ensino e aprendizagem (campo dois), as ideias centrais e o DSC da avaliação dos estudantes de medicina da $4^{a}$ série foram estruturados considerando as respostas sobre a mobilização de recursos, a articulação entre os cenários de aprendizagem e a fidelidade ao método no processo tutorial estabelecido pela instituição e que constituem esse campo do formato de avaliação.

Pergunta 1: A forma como esta Unidade Educacional Sistematizada foi organizada contribuiu para que você mobilizasse os recursos cognitivos, afetivos e psicomotores para desenvolver o cuidado em saúde? Justifique.

IC 1: Impetrou os recursos de aprendizagem - DSC: houve mobilização dos recursos afetivos, cognitivos e psicomotores. (E8, E3)

IC2: Reflexões clínicas e terapêuticas - DSC: Sim, os casos permitiram boas discussões clínicas e terapêuticas. O raciocínio clínico tem sido desenvolvido de forma eficaz e completa. (E1, E3)

Pergunta 2: A forma como esta Unidade Educacional Sistematizada foi organizada contribuiu para a articulação com a Unidade de Prática Profissional no cenário real (UPP)? Justifique

IC1: Correlação teórica e prática eficiente e eficaz - DSC: Muito dos conhecimentos adquiridos na UES puderam ser utilizados na prática. Eles foram eficientes e coerentes nessa articulação. Contribuíram para o desenvolvimento do raciocínio inter-relacionando UPP/LPP e reforçaram o conhecimento teórico aplicado à prática, tendo em vista que os cenários eram cada vez mais reais e exigentes. (E1, E3, E9, E11, E12)

IC2: Teoria e prática às vezes desafinadas - Os casos discutidos na UES nem sempre combinavam com o atual estágio na UPP. Não houve associação da UES com a UPP, salvo em alguns problemas. (E8, E10) 
Pergunta 3: No processo pedagógico, os passos de tutoria estão sendo seguidos?

IC1: Fidelidade aos passos da tutoria - DSC: Os passos do processo tutorial estão sendo seguidos corretamente. Os tutores deste ano são muito bem capacitados e orientados para seguir os passos tutoriais de forma efetiva e didática. (E8, E3)

IC2: Falta preparo do preceptor e tutor- DSC: O tutor discute e o estudante escuta. Não houve casos suficientes para preparar o estudante para o internato. Os professores não explicam os casos, apenas passam a conduta. Na minha tutoria, apenas o tutor discutia, a gente discutia muito pouco, apenas o escutávamos. (E5, E4)

Recursos Educacionais: Ao abordarem os recursos educacionais utilizados para a aprendizagem na Famema, os estudantes manifestaram suas fortalezas e fragilidades, como solicitado no Formato de avaliação (F5).

Indique quais recursos educacionais você não os tenha utilizado ou deles participado nessa unidade: (biblioteca, morfofuncional, consultorias e/ou outros e conferências). Esses recursos e atividades contribuíram para a sua aprendizagem? Justifique. Indique as fortalezas e fragilidades. Caso você não tenha utilizado ou participado, justifique:

IC1: Fortalezas dos recursos educacionais - DSC: A consultoria contribuiu para o esclarecimento de dúvidas essenciais e eventuais e facilitou o aprendizado. Há professores disponíveis em algumas disciplinas e eles trazem suas experiências. A biblioteca contribui para o acesso a fontes de pesquisa que auxiliam no estudo. Os livros são novos e atualizados e os funcionários qualificados. Podem ser utilizados os livros que estão melhor organizados e o serviço de xérox. A biblioteca é simples, mas o ambiente é confortável com bom espaço físico. Por meio dela pude consultar os livros necessários para os problemas abordados na tutoria. Biblioteca disponível é essencial para uma universidade. Ela tem, também, espaço bom para estudos, funcionários atenciosos, acesso a um serviço dos Estados Unidos da América, National Library of Medicine (PUBMED), e outros sites na busca de artigos atuais. Chegaram muitos livros novos e o acesso a periódicos atualizados também é um grande diferencial. A biblioteca e o laboratório morfofuncional contribuíram de forma satisfatória para que o conteúdo sobre medicina aumentasse a nível exponencial e para sedimentar o meu conhecimento. Eles dispõem de recursos, como livros, bonecos, computadores, monitores e outros equipamentos importantes. Essas diversas ferramentas são essenciais para o estudo, pois possuem recursos que simulam o ambiente real. O espaço é sempre aberto aos alunos com recursos de bonecos e materiais para sutura treinando assim o conhecimento teórico na prática (E8, E10, E7, E3, E6) (Higa et al., 2017).

IC2: Fragilidades dos recursos educacionais - DSC: A biblioteca ainda possui muitos livros desatualizados e os atualizados se encontram em número reduzido, com poucos exemplares. Nem sempre é possível ter acesso a alguns exemplares, devido à 
Higa, Moreira, Pinheiro, Tonhom, Carvalho \& Braccialli: Caminhos da avaliação da aprendizagem ativa

quantidade insuficiente de livros para todos os alunos. O xérox nem sempre funciona. Além de faltarem livros para todos os estudantes, também o prazo de devolução é muito pequeno. Poderia haver um sistema de renovação dos livros online. Nas consultorias, muitos professores nos mandam estudar em vez de sanarem nossas dúvidas. Não houve consultoria, pois, o tempo entre a solicitação e o agendamento é grande, o que dificulta porque os casos mudam semanalmente. Em algumas disciplinas, os professores estão indisponíveis. As conferências 7, 8, 11 e 13 foram insatisfatórias, sem qualificação (E8, E2, E6, E10, E5, E 3) (Higa et al., 2017).

\section{Análise de resultados}

Considerando os itens avaliados por meio do F5, referentes ao processo de ensino-aprendizagem, na questão um, que aborda a mobilização dos recursos afetivos, cognitivos e psicomotores para construção do conhecimento, houve consenso positivo entre os estudantes.

Quanto ao posicionamento sobre a articulação entre os cenários da UPP e UES, (questão dois), a maioria aponta que os problemas discutidos na UES articulam com os problemas reais. Infelizmente, a minoria reconhece um descompasso na correlação entre a teoria e a prática.

Em relação à questão três, na qual eles identificam o cumprimento dos passos do processo tutorial, há consensos e dissensos, pois, na mesma proporção, eles abordam que há tutores fiéis ao método e outros que limitam a participação dos estudantes com a discussão centrada apenas em si mesmos.

A literatura assinala que, nesse currículo orientado por competência, com abordagem holística e dialógica para profissionais de saúde, há necessidade de propiciar experiências diversas e reflexivas em cenários reais e simulados ao longo de todo o curso com a intencionalidade de possibilitar articulação dos recursos para o desenvolvimento de ações definidas e validadas na formação médica (Ribeiro, Lima, \& Padilha, 2018).

$\mathrm{Na}$ quarta série do curso médico, a UES se organiza por meio de tutorias, conferências, laboratório de parasitologia, curso de radiologia e, ainda, há realização de trabalho científico com professor orientador, além da Unidade de Prática Profissional (Faculdade de Medicina de Marília, 2016). As DCN de 2014 indicam que a formação médica deve privilegiar e articular diferentes cenários de aprendizagem, tendo em vista sua formação em atenção, gestão e educação à saúde, dentre outros requisitos (Brasil, 2014).

Considerando o que se preconiza para a construção do conhecimento na estrutura curricular da Famema, pela representação social do estudante de medicina da $4^{a}$ série, o planejamento da UES contribui positivamente para sua formação. Estudos realizados na Famema por meio do Formato Cinco de avaliação (F5) sobre o planejamento 
curricular, em todos os cenários de aprendizagem, vêm demonstrando a importância dessa organização para a formação em saúde e confirmam esse posicionamento dos estudantes também sobre a UES (Carvalho, Taipeiro, Higa, Aguilar-da-Silva, Tonhom, \& Hafner, 2016; Castilho et al., 2017; Higa, Taipeiro, Aguilar-da-Silva, Tonhom, Carvalho \& Hafner, 2016; Higa et al., 2017).

De modo geral, ainda que haja necessidade de pequenos ajustes no processo de ensino e aprendizagem, o estudante, em seu protagonismo, vem apontando, nas avaliações, os benefícios do planejamento educacional para o desenvolvimento da competência requerida no trabalho em saúde, para que ele possa se inserir no mercado de trabalho e promover a transformação da realidade do cuidado (Higa et al., 2017).

Em vista disso, há de se compreender a importância do papel do professor na lógica de organização da aprendizagem ativa para que possa efetivamente caminhar de uma estrutura centrada no conteúdo e no professor para o papel de mediador, estimulador e inovador da aprendizagem. É necessário, portanto, revisitar o paradigma educacional, refletir sobre sua aplicabilidade no momento e construir conjuntamente outras possibilidades. Nesse âmbito, deve ser estabelecida uma sólida relação do professor com o estudante na construção do conhecimento e no processo avaliativo (Diesel, Baldez, \& Martins, 2017; Farias, Martin, \& Cristo, 2015; Higa et al., 2017).

Desta forma, verifica-se que a avaliação da aprendizagem ativa valoriza permanentemente a devolutiva ao estudante sobre suas conquistas e necessidades de avanços e oportuniza a construção de significados e o desejo de novos saberes. Nesse processo, professores e estudantes se corresponsabilizam pela avaliação e progressão da aprendizagem. Essa perspectiva de avaliação almeja superar o paradigma tradicional, que enfoca o sucesso e o fracasso, a culpabilização e o medo da falha, resultados generalizados com tempo igualmente determinado para todos, cujo único parâmetro é a visão do avaliador (Gomes, Petta \& Pereira, 2018).

Os estudantes apontaram ainda, a importância dos recursos educacionais em metodologias ativas, bem como o que precisa ser melhorado para sua plena aprendizagem.

Sobre esses recursos, a instituição, em seu planejamento da quarta série, preconiza que a biblioteca possua literatura básica para formação médica e facilite o acesso às bases confiáveis para construção do conhecimento. A consultoria contempla esse processo e o laboratório morfofuncional disponibiliza os recursos complementares para a aprendizagem (Faculdade de Medicina de Marília, 2016).

Esta participação do estudante no processo avaliativo é preconizada no Manual de Avaliação que a caracteriza como um contínuo de aprendizagem. Desse modo, contribui com a transformação humana e profissional e busca desmistificar o paradigma tradicional de "acerto de contas" e competição entre estudantes e professores (Faculdade de Medicina de Marília, 2015; Higa et al., 2017). 
Higa, Moreira, Pinheiro, Tonhom, Carvalho \& Braccialli: Caminhos da avaliação da aprendizagem ativa

$\mathrm{Na}$ aprendizagem ativa, o estudante representa a figura central da ação educativa e o planejamento da estrutura institucional deve ser pensado e considerado de modo que favoreça sua autonomia, com recursos e espaços colaborativos, estimuladores e facilitadores da aprendizagem. Essa interação humana constante pode contribuir para o estudante pensar sobre as situações cotidianas e desenvolver seu senso crítico e reflexivo (Diesel, Baldez, \& Martins, 2017; Higa et al., 2017; Higa et al., 2017).

\section{Conclusões}

A representação social dos estudantes sobre o planejamento do processo de ensino e aprendizagem desenvolvido na UES indica que ele contribui para mobilização dos recursos necessários ao desenvolvimento da competência profissional e desenvolve o raciocínio clínico para o cuidado. A maioria dos participantes refere uma boa correlação teórico prática, enquanto alguns apontam a necessidade de adequações, bem como melhor preparo de professores responsáveis pela formação médica.

Considerando o objetivo desta pesquisa, foi possível reconhecer e compreender os aspectos relacionados ao processo de aprendizagem e aos recursos educacionais, os quais sinalizaram a positividade na articulação entre os recursos afetivos, psicomotores e cognitivos, momentos de articulação entre a teoria e a prática e acesso aos recursos educacionais na construção de novos conhecimentos.

Os estudantes apontaram ainda, a necessidade de melhorias fundamentais em toda a estrutura. Essas envolvem a capacitação dos docentes no exercício de sua função, passam pela adequação e ajuste no compasso entre a teoria e a prática, revisão dos conteúdos das conferências, complementação dos recursos institucionais faltantes ou precários, bem como a reestruturação das consultorias. Acrescentaram, também, sugestões de melhorias no formato de avaliação.

Esses resultados podem subsidiar a tomada de decisão institucional, tendo em vista a continuidade do desenvolvimento curricular e da avaliação formativa instituída no caderno de planejamento da aprendizagem da quarta série e no projeto pedagógico do Curso de Medicina da Famema, como preconizados pelas DCN e diretrizes do SUS em nosso país.

Assim sendo, esses aspectos merecem reflexões dos gestores, docentes e estudantes, tendo em vista a compreensão dos envolvidos e os ajustes que se fizerem necessários.

Vale destacar que os pressupostos da pesquisa qualitativa permitiram, por meio da Teoria das Representações Sociais e da técnica de análise do Discurso do Sujeito Coletivo, fotografar a realidade em que se refletiu a boa e importante estrutura organizacional da UES, bem como a necessidade de adequações e avanços que caminhem para as melhorias do processo de ensino e aprendizagem. 


\section{Referências}

Antunes, A. P. (2017). Formação académica em metodologia qualitativa: Prática pedagógica em Psicologia da Educação. Revista Lusófona de Educação, 36, 147-161.

Barrows HS, Tamblyn RM (1980). Problem-based learning: an approach to medical education. New York: Springer.

Bettencourt, M. B., \& Sá Earp, M. L. (2017). Significados das avaliações do ensino pelos estudantes: um estudo qualitativo na educação superior brasileira. Revista Lusófona de Educação, 36, 131-145.

Brasil. (2013). Ministério da Saúde. Resolução n. 466, de 12 de dezembro de 2012. Aprova as diretrizes de normas regulamentadoras de pesquisas envolvendo seres humanos. Brasília: Diário Oficial da União.

Brasil. (2014). Ministério da Educação. Resolução CNE/CES n. 3, de 20 de junho de 2014. Institui Diretrizes Curriculares Nacionais do Curso de Graduação em Medicina e dá outras providências. Brasília: Diário Oficial da União.

Carvalho, M. H. R., Taipeiro, E. F., Higa, E. F. R., Aguilar-da-Silva, R. H., Tonhom, S. F. R., Pinheiro, O. L., \& Hafner, M. de L. B. (2016). A visão do estudante no mundo do trabalho: avaliação da Unidade de Prática Profissional. Revista Saúde em Redes, 2 (1 supl), 946.

Castilho, A. R., Higa, E. F. R., Otani, M. A. P., \& Soares, M. O. M. (2017). Evaluation of active learning in the vision of nursing students. Journal of Nursing UFPE on Line, 11(2 supl), 973-983. [Disponível em https://periodicos.ufpe. br/revistas/ revistaenfermagem/article/view/13467/0, consultado em 20/12/2017].

Diesel, A., Baldez, A. L. S., \& Martins, S. N. (2017). Os princípios das metodologias ativas de ensino: uma abordagem teórica. Revista Thema, 14(1), 268-288.

Duran, M. C. G. (2012). Representações sociais: uma instigante leitura com Moscovici, Jodelet, Marková e Jovchelovitch. Educação \& Linguagem, 15(25), 228-243.

Faculdade de Medicina de Marília. (2014). Projeto pedagógico do curso de medicina. Marília: Faculdade de Medicina de Marília.

Faculdade de Medicina de Marília. (2015). Caderno de avaliação: cursos de medicina e enfermagem. Marília: Faculdade de Medicina de Marília.

Faculdade de Medicina de Marília. (2016). Atenção às necessidades de saúde do indivíduo, família e comunidade, no modelo de vigilância á saúde e Unidade Prática Profissional 4: 4ª série de Medicina. Marília: Faculdade de Medicina de Marília.

Farias, P. A. M., Martin, A. L. A. R., \& Cristo, C. S. (2015). Aprendizagem ativa na educação em saúde: percurso histórico e aplicações. Revista Brasileira de Educação Médica, 39(1), 143-150. [Disponível em http://www.scielo.br/pdf/rbem/v39n1/1981-5271-rbem-39-1-0143.pdf, consultado em 20/12/2017].

Felix, G. T., Bertolin, J. G., \& Polidori, M. M. (2017). Avaliação do ensino superior: um comparativo dos instrumentos de regulação entre Brasil e Portugal. Avaliação, 22(1), 35-54.

Figueiredo, M. Z. A., Chiari, B. M., \& Goulart, B. N. G. (2013). Discurso do Sujeito Coletivo: uma breve introdução à ferramenta de pesquisa qualiquantitativa. Distúrbios da Comunicação, 25(1), 129-136.

Gomes, R. Petta, H. I. L., \& Pereira, S. M. S. F. (2018). Práticas avaliativas: bases conceituais na formação profissional em saúde. In: Reflexões e inovações na educação de profissionais de saúde. Rio de Janeiro, Atheneu, 101-110. 
Higa, Moreira, Pinheiro, Tonhom, Carvalho \& Braccialli: Caminhos da avaliação da aprendizagem ativa

Higa, E. F. R., Moreira, H. M., Pinheiro, O. L., Tonhom, S. F. R., Carvalho, M. H. R., \& Braccialli, L. A. D. (2017). Avaliação do processo e dos recursos educacionais na visão do estudante de medicina. In A. P. Costa, J. Ribeiro, E. Synthia, \& D. N. Souza (Eds.), CIAIQ 2017 (Vol. 1, pp. 72-80). Salamanca: Ludomedia. [Disponível em http://proceedings.ciaiq.org/index.php/ ciaiq2017/article/ view/ 1320/1278, consultado em 20/12/2017].

Higa, E. F. R., Taipeiro, E. F., Carvalho, M. H. R., Aguilar-da-Silva, R. H., Tonhom, S. F. R., \& Hafner, M. L. M. B. (2017). Avaliação da aprendizagem ativa na graduação em saúde. Revista San Gregorio, (16), 60-69. [Disponível em http://revista.sangregorio.edu.ec/index.php/REVISTASANGREGORIO/ article/view/420/SEIS, consultado em 20/12/2017].

Lefèvre, F., \& Lefèvre, A. M. C. (2005a). Depoimentos e discursos: uma proposta de análise em pesquisa social (Vol. 12). Brasília: Líber Livro.

Lefèvre, F., \& Lefèvre, A. M. C. (2005b). O discurso do sujeito coletivo: um novo enfoque em pesquisa qualitativa. Caxias do Sul: EDUCS. Caxias do Sul: EDUCS.

Lefèvre, F., \& Lefèvre, A. M. C. (2012). Pesquisa de representação social: um enfoque qualiquantitativo: a metodologia do discurso do sujeito coletivo. Brasília: Líber Livro.

Leon, L. B., \& Onófrio, F. Q. (2015). Aprendizagem Baseada em Problemas na graduação médica: uma revisão da literatura atual. Revista Brasileira de Educação Médica, 39(4), 614-619. [Disponível em http://www.scielo.br/pdf/rbem/v39n4 /1981-5271-rbem-39-4-0614.pdf, consultado em 15/12/2017].

Moscovici, S. (2009). Representações sociais: investigações em psicologia social. Petropolis: Vozes.

Reis, S. L. A., \& Bellini, L. M. (2013). Representações sociais como teoria e instrumento metodológico para a pesquisa em educação ambiental. Reflexão E Ação, 21(1), 276-294. [Disponível em https:// online.unisc.br/seer/index. php/reflex/article/view/1440/2920, consultado em 12/03/2016].

Ribeiro, E. C. O., Lima,V. V., \& Padilha, R. Q. (2018). Formação orientada por competência. In: Reflexões e inovações na educação de profissionais de saúde. (pp. 25-36).Rio de Janeiro:Atheneu.

Elza de Fátima Ribeiro Higa

Docente da Graduação e Pós-Graduação Lato e Stricto Sensu e do Grupo de

Avaliação

Coordenadora do Núcleo de Desenvolvimento Docente

Chefe da Disciplina de Educação em Ciências da Saúde

Faculdade de Medicina de Marília - Famema, Marília, São Paulo, Brasil

Email: hirifael@gmail.com ORCID:0000-0001-5272-9597

Haydée Maria Moreira

Docente da Graduação Disciplina de Fisiologia Coordenadora do Núcleo de Avaliação Institucional Faculdade de Medicina de Marília - Famema, Marília, São Paulo, Brasil Email: haydee33@gmail.com ORCID:0000-0001-6866-7133 
Osni Lázaro Pinheiro

Docente da Graduação e Pós-Graduação Lato e Stricto Sensu e do Grupo de

Avaliação

Coordenador do Mestrado Profissional "Ensino em Saúde"

Disciplina de Farmacologia e Terapêutica Experimental

Faculdade de Medicina de Marília - Famema, Marília, São Paulo, Brasil

Email: osnilp100@gmail.com

ORCID: 0000-0002-6533-0428

Silvia Franco da Rocha Tonhom

Docente da Graduação e Pós-Graduação Lato e Stricto Sensu e do Grupo de

Avaliação

Chefe da Disciplina de Enfermagem em Saúde Coletiva Faculdade de Medicina de Marília - Famema, Marília, São Paulo, Brasil

Email: siltonhom@gmail.com ORCID: 0000-0001-7522-2861

Maria Helena Ribeiro de Carvalho

Docente da Graduação e do Grupo de Avaliação Disciplina Enfermagem Pediátrica

Faculdade de Medicina de Marília - Famema, Marília, São Paulo, Brasil

Email: marihel2002@uol.com

ORCID: 0000-0002-0680-9711

Luzmarina Aparecida Doretto Braccialli

Docente da Graduação e Pós-Graduação Lato e Stricto Sensu e do Grupo de

Avaliação

Disciplina Enfermagem em Saúde Coletiva

Faculdade de Medicina de Marília - Famema, Marília, São Paulo, Brasil

Email: luzbra@terra.com.br

ORCID: 0000 -0002-9622-8629

Correspondência

Elza de Fátima Ribeiro Higa

Avenida José de Grande, n. 332, Jardim Parati - CEP - 17519470

Faculdade de Medicina de Marília - Famema, Marília, São Paulo, Brasil

Data de submissão: Março de 2017

Data de avaliação: Abril de 2017

Data de publicação: Julho 2018 